\title{
Does negative expiratory pressure influence performances of spirometry in older patients?
}

\author{
D. Vanpee*, Ch. Swine ${ }^{\#}$, J.P. Delwiche ${ }^{\Uparrow}$, J. Jamart ${ }^{+}$, L. Delaunois
}

Does negative expiratory pressure influence performances of spirometry in older patients? D. Vanpee, Ch. Swine, J.P. Delwiche, J. Jamart, L. Delaunois. (C)ERS Journals Ltd 2002.

ABSTRACT: The aim of this study is to assess the influence of the negative expiratory pressure (NEP) technique on the performance of maximal expiratory manoeuvre in elderly patients.

Firstly, the authors studied how NEP (at 5 and $10 \mathrm{cmH}_{2} \mathrm{O}$, NEP5 and NEP10) influences forced vital capacity (FVC) and forced expiratory volume in one second (FEV1) in 60 young healthy volunteers, in order to assess the fluctuations of the method. In the second part of the study, 65 successive elderly inpatients ( $>70$ yrs old) were examined. In this group, 15 elderly patients were unable to perform the manoeuvre, 27 had a normal function, 14 had a lung function with obstructive pattern and nine with restrictive pattern.

In young subjects, FVC during NEP5 and NEP10 compared to baseline values was $101 \pm 5 \%$ and $103 \pm 5 \%$, respectively, and FEV1 was $100 \pm 4 \%$ and $101 \pm 5 \%$, respectively. In elderly patients with normal function, FVC during NEP5 and NEP10 compared to baseline values was $99 \pm 14 \%$ and $109 \pm 23 \%$, respectively, and FEV1 was $97 \pm 9 \%$ and $104 \pm 13 \%$, respectively. There were no relevant effects of the NEP application on FVC or FEV1 values in elderly patients with an obstructive or restrictive pattern.

In elderly patients, the use of the negative expiratory pressure technique during maximal expiratory manoeuvres provides little complementary information compared to a classical manoeuvre. The negative expiratory pressure technique did not modify the initial diagnosis when compared with the classical manoeuvre.

Eur Respir J 2002; 20: 674-678.

\begin{abstract}
*Depts of Emergençy Medicine, ${ }^{\#}$ Geriatric Medicine, Pneumology and Biostatistics, Université Catholique de Louvain, Mont-Godinne Hospital, Yvoir, Belgium.
\end{abstract}

Correspondence: D. Vanpee

Emergency Unit

Mont-Godinne Hospital

5530 Yvoir

Belgium

Fax: 3281423115

E-mail: Dominique.Vanpee@

rean.ucl.ac.be

\section{Keywords: Elderly}

maximal expiratory manoeuvres

negative expiratory pressure technique

Received: November 212001

Accepted after revision: April 182002
Forced expiratory manoeuvres including forced expiratory volume in one second (FEV1) are routinely used to assess the maximal expiratory flows that a subject can achieve at different lung volumes. The results are strongly dependent on the cooperation of the patient. One of the largest sources of within-subject variability of forced expiratory manoeuvres is performance of the test with insufficient effort to reach the expiratory flow limitation (FL). This lack of collaboration is particularly present in elderly patients. In the past, no method has been available to assess whether an expiratory FL was reached during the forced expiratory manoeuvre. Recently, however, VolTA et al. [1] showed that applying the negative expiratory pressure (NEP) technique during forced vital capacity (FVC) in normal adults is a good method for assessing flow limitation [1]. This technique for assessing whether maximal flows are obtained during a forced manoeuvre has the potential to improve the assessment of pulmonary function in elderly subjects.

The application of an NEP at the airway opening during the forced expiratory manoeuvre increases the driving pressure for flow. If flow increases with the application of NEP, then FL is not present. Conversely, if there is no increase in flow with NEP, then FL has been achieved.
The NEP method has been previously used to assess the performance of the FVC manoeuvre in children [2] and normal adults [1]. The aim of this study is to assess the influence of the NEP technique on the performance of maximal expiratory manoeuvre in elderly patients.

\section{Materials and methods}

Before studying the influence of the NEP technique in elderly patients, an understanding of the fluctuation of the method in normal subjects was needed. Initially, therefore, the authors studied the influence of NEP on FVC and FEV1 in 60 young normal healthy volunteers during forced expiratory manoeuvre.

The study was approved by the institutional ethics Committee.

\section{Population}

First part of the study. Sixty healthy nonsmoking subjects ( 27 males and 33 females) without previous history of cardiopulmonary disease were studied. The mean age of the group was $33 \pm 10 \mathrm{yrs}$, with a mean 
Table 1. - Anthropometric characteristics and lung function data

\begin{tabular}{lccccccc}
\hline & $\begin{array}{c}\text { Subject } \\
\mathrm{n}\end{array}$ & $\begin{array}{c}\text { Age } \\
\text { yrs }\end{array}$ & $\begin{array}{c}\text { Height } \\
\mathrm{cm}\end{array}$ & $\begin{array}{c}\text { Weight } \\
\mathrm{kg}\end{array}$ & $\begin{array}{c}\text { FVC } \\
\% \text { pred }\end{array}$ & $\begin{array}{c}\text { FEV1 } \\
\% \text { pred }\end{array}$ & $\begin{array}{c}\text { FEV1/FVC } \\
\%\end{array}$ \\
\hline Healthy subjects & 60 & $33 \pm 10$ & $171 \pm 10$ & $69 \pm 16$ & $118 \pm 19$ & $118 \pm 19$ & $83 \pm 8$ \\
Normal elderly subjects & 27 & $82 \pm 5$ & $160 \pm 9$ & $66 \pm 13$ & $99 \pm 21$ & $104 \pm 18$ & $79 \pm 12$ \\
Obstructive elderly subjects & 14 & $78 \pm 5$ & $164 \pm 7$ & $64 \pm 13$ & $77 \pm 19$ & $57 \pm 16$ & $55 \pm 8$ \\
Restrictive elderly subjects & 9 & $83 \pm 7$ & $165 \pm 4$ & $64 \pm 11$ & $56 \pm 9$ & $60 \pm 10$ & $79 \pm 6$ \\
\hline
\end{tabular}

Data are presented as mean $\pm \mathrm{SD}$ of the European Respiratory Society predicted values. FVC: forced vital capacity; FEV1: forced expiratory volume in one second; \% pred: $\%$ predicted.

weight of $69 \pm 16 \mathrm{~kg}$ and mean height of $171 \pm 10 \mathrm{~cm}$. Their anthropometric characteristics and lung function data are given in table 1.

Second part of the study. The second part of the study examined 65 successive elderly inpatients ( $>70 \mathrm{yrs}$ old, 21 males and 44 females) from a geriatric unit. Among them, 15 were unable to correctly perform the FVC manoeuvre. The other 50 were divided into three groups according to values of lung function data (table 1). Patients with an FEV1 $>80 \%$ of European Respiratory Society (ERS) predicted values [3] were considered normal. Patients with an FEV $1<80 \%$ and with an $\mathrm{FEV} 1 / \mathrm{FVC}<70 \%$ were considered "obstructive". Patients with an FEV $1<80 \%$ but with an FEV1/ FVC $>70 \%$ were considered "restrictive".

\section{Pulmonary function testing}

One of the authors, a board certified pneumologist, administered all spirometry testings. Subjects were instructed about the FVC manoeuvre according to the American Thoracic Society (ATS) standard [4], and the technique was demonstrated several times if necessary just before testing. Because flow during the FVC manoeuvre depends on the pattern of the preceding inspiration [5], the subjects were asked to perform FVC manoeuvres after a standardised previous time history, which consisted of a maximal inspiration from functional residual capacity lasting $\pm 2 \mathrm{~s}$, followed directly by the expiratory manoeuvre. For those subjects who had difficulty performing the FVC manoeuvre, repeated explanations and demonstrations were given after each test, based upon the problems identified with the previous manoeuvre. Each participant was asked to try to perform the forced expiratory manoeuvre in order to obtain three acceptable manoeuvres. The pneumotachograph was calibrated just before testing each subject. The largest FEV1 and FVC, corrected to body temperature and ambient pressure, and saturated with water vapour (BTPS), were converted into percentage of predicted values [3]. Subjects were only included if they were able to perform a minimum of three acceptable FVC manoeuvres. Acceptability was defined using the ATS guidelines. Reproducibility was defined as present if at least two of the FVC and FEV1 out of the three acceptable tracings did not vary by $>5 \%$ [4].

A flanged plastic mouthpiece was connected in series with a pneumotachograph and a circular Venturi device (Micro 5000; Medisoft, Dinant, Belgium) capable of generating a negative pressure during expiration. One end of the device was the opening to the atmosphere. The other end was connected to the external cone of the pneumotachograph. A side opening on the Venturi device was connected via an electrically operated solenoid valve to a cylinder of compressed air. The solenoid valve was controlled by a computer. The Venturi device was attached downstream of the pneumotachograph and was set to produce an airway pressure of -5 or $-10 \mathrm{cmH}_{2} \mathrm{O}$ controlled by a manometer. NEP was applied to the airway opening early in the forced expiratory manoeuvre (when an expiratory flow of $0.03 \mathrm{~L} \cdot \mathrm{s}^{-1}$ was achieved) and maintained until the forced manoeuvre was complete (long-pulse method). The effective appliance of the pressure can be checked simultaneously, as shown on figure 1 .

Patients were studied sitting comfortably on a chair and wearing nose clips. They were instructed to fully inspire from functional residual capacity after a warning signal and then expire as fast and hard as possible. The subjects performed three series of three FVC manoeuvres, one without NEP (baseline), and, in random order, one with NEP $-5 \mathrm{cmH}_{2} \mathrm{O}$ and another with NEP $-10 \mathrm{cmH}_{2} \mathrm{O}$ (NEP5 and NEP10). The manoeuvres were realised at a frequency of one every 2 min. Testing was repeated until three acceptable manoeuvres were obtained. The following parameters were analysed: FVC, FEV1, FEV1/FVC.

Results are given as \% predicted values or $\%$ of baseline values and are expressed as mean \pm SD. Reference ranges are estimated by 2.5 and 97.5 percentiles from a normal distribution. The parameters were compared by paired or impaired t-tests as appropriate.

\section{Results}

\section{Healthy subjects}

In the 60 young healthy subjects, FVC during NEP5 and NEP10 compared to baseline values was $101 \pm 5$ (nonsignificant $(\mathrm{NS})$ ) and $103 \pm 5 \%(\mathrm{p}<0.001)$, respectively. The difference between NEP5 and NEP10 was significant $(\mathrm{p}<0.001)$. Values ranged from 91$111 \%$ and $93-114 \%$.

FEV1 during NEP5 and NEP10 compared to baseline values was $100 \pm 4 \%$ (NS) and $101 \pm 5 \%$ (NS), respectively. The values for NEP5 and NEP10 did not differ significantly. Values ranged from $92-108 \%$ and $91-111 \%$. 


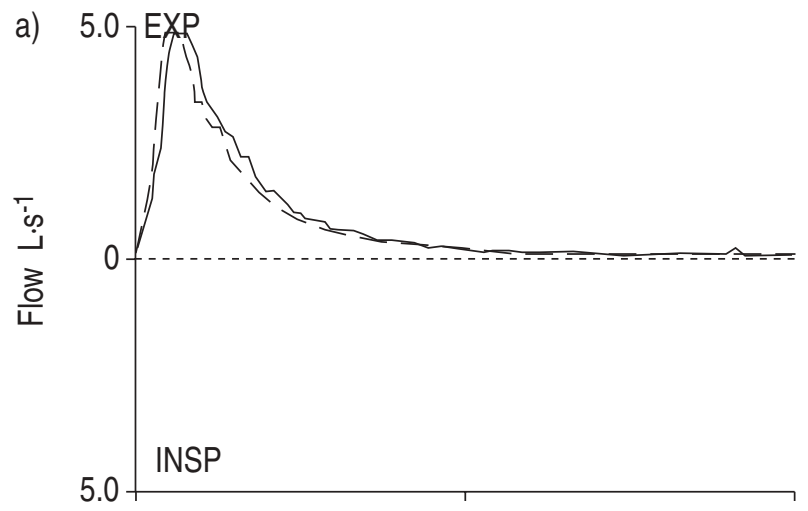

b)

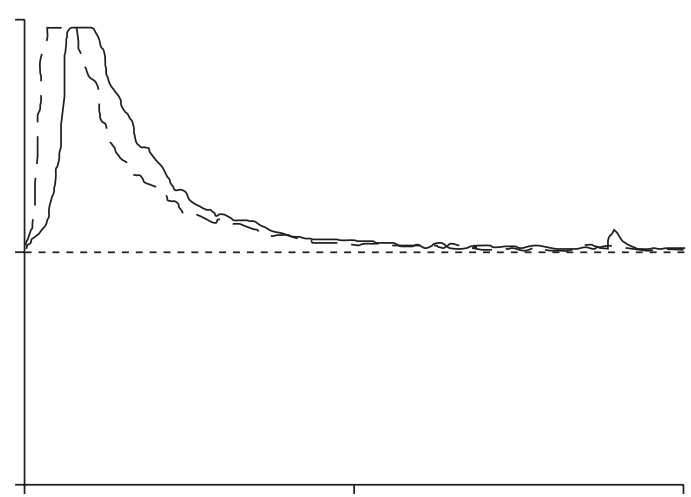

d)

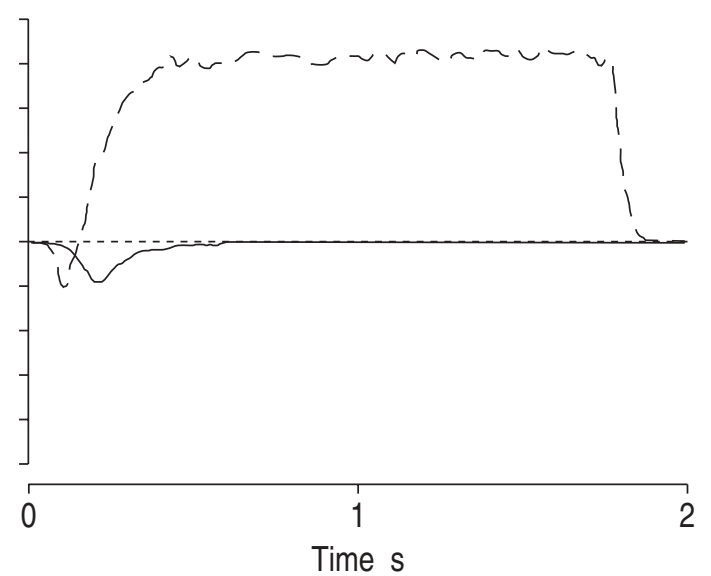

Fig. 1.-Expiratory flow according to time during control (-): a) negative expiratory pressure (NEP) 5 and b) NEP10 manoeuvres (---). Mouth pressure $(P \mathrm{~m})$ according to time during control $(-)$ : c) NEP5 and d) NEP10 (-- $)$ manoeuvres. The NEP pressure is applied when the expiratory flow reaches $0.030 \mathrm{~L} \cdot \mathrm{s}^{-1}$. The apparent delay for $P_{\mathrm{m}}$ to reach the maximal expiratory value is due to the cumulative effects of the positive expiratory pressure caused by the forced expiration in the tube and the negative pressure of the Venturi system (Micro 5000; Medisoft) inducing NEP. EXP: expiratory; INSP: inspiratory.

The flow/volume curves obtained with and without NEP were visually the same in all cases. NEP was well tolerated by all subjects.

\section{Elderly subjects}

Among the 65 elderly patients, 15 were unable to perform the forced expiratory manoeuvre because of poor coordination or cognitive disturbance. According to the criteria described above, 27 elderly patients had a normal function, 14 had lung function of obstructive pattern and nine of restrictive pattern. The anthropometric characteristics and lung function data of each group are given in table 1 .

Elderly patients with normal function. FVC during NEP5 and NEP10 compared to baseline values was $99 \pm 14 \%$ (Ns) and $109 \pm 23 \%(\mathrm{p}=0.05)$, respectively. The difference between NEP5 and NEP10 was significant $(\mathrm{p}=0.003)$. Values ranged from $71-127 \%$ and $64-154 \%$.

FEV1 during NEP5 and NEP10 compared to baseline values was $97 \pm 9 \%(\mathrm{p}=0.05)$ and $104 \pm 13 \%$ (NS), respectively. The difference between NEP5 and
NEP10 was significant $(\mathrm{p}=0.005)$. Values ranged from $79-114 \%$ and $78-130 \%$.

When each patient was studied separately, it was noted that during NEP5, FVC increased $>20 \%$ in three cases $(20,24,43 \%$ of baseline values, respectively), without significant increase of FEV1. During NEP10, FVC increased $>20 \%$ in four cases the same three as NEP5 with one other) $(27,47,61$ and $76 \%$, respectively). All these patients had a control FVC lower than expected (predicted FVC not obtained) and corrected to predicted values during NEP10, means of a prolonged expiration (fig. 2).

Elderly patients with obstructive pattern. Fourteen elderly patients had an obstructive pattern (see table 1).

FVC during NEP5 and NEP10 compared to baseline values was $93 \pm 12 \%$ (Ns) and $100 \pm 14 \%$ (Ns), respectively. The difference between NEP5 and NEP10 was significant $(\mathrm{p}=0.041)$.

FEV1 during NEP5 and NEP10 compared to baseline values was $100 \pm 13 \%$ (NS) and $106 \pm 12 \%$ $(\mathrm{p}=0.065)$, respectively. FEV1 for NEP5 and NEP10 did not differ significantly.

When each patient was analysed separately, none 

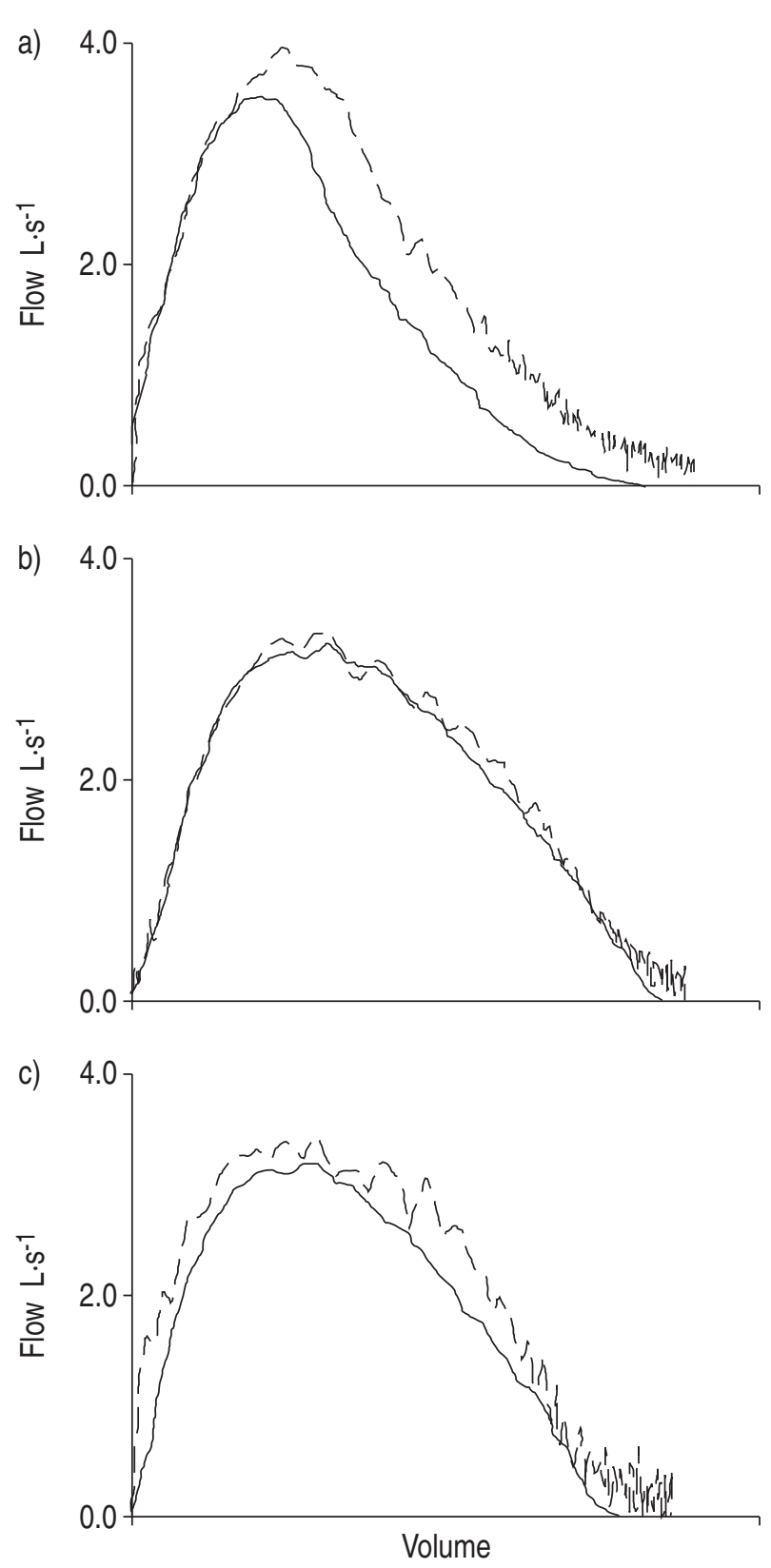

Fig. 2. - Flow/volume curves in control (-) and a) under negative expiratory pressure (NEP) 5 (--) conditions in patient 1 with an increase of the expiratory flow and forced vital capacity (FVC), b) under NEP5 (--) conditions in patient 2 who did not increase flow and FVC, and c) the same patient's increasing flow and FVC at NEP10 (-- ).

of the patients' FEV1 improved $>20 \%$ during NEP5. During NEP10, the FEV1 of two patients improved $>20 \%$ (20 and $29 \%$, respectively). This increase of FEV1 was higher than the SD obtained in the normal elderly patients during NEP10. Despite this increase, both FEV1 values remained $<80 \%$ pred and the diagnosis of obstruction was not modified.

Elderly patients with restrictive pattern. Nine elderly patients had a restrictive pattern (table 1).

FVC during NEP5 and NEP10 compared to their baseline values was $101 \pm 19 \%$ (NS) and $110 \pm 16 \%$ $(\mathrm{p}=0.089)$, respectively. The difference between NEP5 and NEP10 was significant $(\mathrm{p}=0.023)$.

FEV1 during NEP5 and NEP10 compared to baseline values was $97 \pm 18 \%$ (Ns) and $100 \pm 17 \%$ (NS), respectively. The difference between NEP5 and NEP10 was not significant.

When each patient was analysed separately, it was observed that with NEP5, only one patient had an improved $\mathrm{FVC}>20 \%$ (25\%). During NEP10, four patients had an improved FVC $>20 \%(20,24,25$ and $31 \%$, respectively) by prolonging their expiratory time. Despite this significant increase, FVC values remained $<80 \%$ of pred and the diagnosis of pulmonary restriction was not modified.

\section{Discussion}

The FVC manoeuvre is used to assess the FEV1 and maximal flows that a subject can achieve at different lung volumes. Forced expiratory manoeuvres are routinely used to assess lung function in elderly patients. Neither age nor obstructive disease is incompatible with highly reproducible spirometric tests on elderly patients, provided a rigorous quality control programme is established [6]. However, in patients with cognitive impairment it is not always possible. A study reported that eight (12.3\%) of 65 outpatients $>65$ yrs old could not perform three acceptable FVCs, and scored lower on tests of psychomotor speed and executive function [7]. In another study, in 208 institutionalised subjects characterised by old age $(83.7 \pm 8.2)$ and severe deterioration, only $40 \%$ were able to perform spirometry. The poorer performance was seen in subjects with lower mini mental score evaluation (MMSE) scores [8]. Similarly in the present study, 15 out of 65 were unable to perform technically satisfactory FVC manoeuvres because of cognitive impairment, poor coordination and lack of cooperation.

In the elderly population, the assessment and interpretation of pulmonary function are not always easy in clinical practice. Good cooperation of the patient is indispensable in the performance of classical forced expiratory manoeuvres. Therefore, the current authors wanted to know whether the NEP technique would change the results and interpretation of the forced expiratory manoeuvres. In fact, the application of an NEP to the mouth during these manoeuvres could perhaps help some patients to achieve FL or sustain effort until residual volume is reached.

The main findings of the present study are as follows. 1) In the group of young subjects, a significant but not relevant increase of the mean FVC during NEP10 (3\%) was observed, although a larger increase $(\leqslant 13 \%)$ was found in some patients. This was not observed during NEP5. The mean FEV1 did not change with the two levels of NEP. Therefore, the application of an NEP in healthy young subjects has very little influence on the mean FVC and FEV1, although some individuals can show increase of $\leqslant 13 \%$. The reference values for FEV1 and FVC from recent studies have presented higher values than 
those used in this study [3], which could explain why the control values of the young healthy subjects in this study were $118 \%$ pred [9]. Most of these subjects probably achieved FL during control measurements. 2) In the elderly normal subjects, even if the fluctuation is more important $(\leqslant 53 \%$ in some individuals), it was observed that the application of NEP during a forced expiratory manoeuvre weakly influenced the mean values of the FVC $(9 \%)$ and FEV1 $(4 \%)$. The control values of the normal elderly subjects were $99-104 \%$ pred [3] and the small improvement that was observed could have been due to both a better and longer expiration and the fact that the values predicted by QUANJER et al. [3] may slightly underestimate the reference values [9]. 3) In the elderly obstructive group, the application of an NEP5 and NEP10 had no significant or relevant influence on the FVC or FEV1. In this group, only two patients experienced a significant improvement in FEV1 during NEP10, but even if this increase was higher than the SD obtained in the normal elderly patients, the values of FEV1 remained $<80 \%$ pred. Therefore, application of an NEP during maximal expiratory manoeuvre did not modify the interpretation of the diagnosis of obstruction. 4) In the elderly patient group with restrictive pattern, the mean FVC values during NEP5 and NEP10 did not change significantly. In this group, four patients experienced an improvement in FVC during NEP10, but despite this increase, FVC values remained $<80 \%$ pred and the diagnosis of pulmonary restriction did not change.

Therefore, the current authors believe that in elderly patients, the use of an NEP technique during maximal expiratory manoeuvre brings little complementary information compared to a classical manoeuvre, despite the fact that the NEP technique was well tolerated and did not present any significant technical difficulty.

In patients unable to perform the maximal expiratory manoeuvre (15 out of 65 in this study), the application of an NEP during resting breathing could perhaps evaluate the FL. Five of the 15 were flow limited during tidal breathing expiration at $-10 \mathrm{cmH}_{2} \mathrm{O}$ [10], but the significance of this obstruction needs to be studied further.

The long pulse method used in this study. The advantage of this technique is that it can assist some patients in achieving FL or sustaining their expiratory effort until residual volume [11]. The fact that the baseline and the NEP curves were the same indicates that most of the patients in this study had already achieved FL during the baseline measurements.

These results differ from these of VoLTA et al. [1], where a small but significant reduction in FVC with NEP10 (long pulse method) was found. This averaged $3.5 \%$ of the control FVC but without significant difference for the other pulmonary function parameters. VOLTA et al. [1] explained the finding by suggesting that at very low lung volumes, NEP10 can elicit collapse of the extrathoracic airways. Their results are not confirmed in this study, which used a much larger population.

In contrast, when NEP was applied in children [11], there was a statistically significant increase in FEV1 with the long pulse NEP, but the mean increase of $<2 \%$ was not clinically relevant. In the paediatric population, the long pulse has some disadvantages, as it is difficult to quantify the change in flow, and the sustained application of NEP until residual volume may result in more frequent leaks near the end of the manoeuvre [11].

In conclusion, in elderly patients, the use of the negative expiratory pressure technique during maximal expiratory manoeuvres provides little complementary information compared to a classical manoeuvre. For elderly patients with normal function, negative expiratory pressure has little influence on the forced vital capacity and the forced expiratory volume in one second. For most obstructive elderly patients, the negative expiratory pressure technique does not improve forced expiratory volume in one second, and with regard to restrictive pattern, it does not improve forced vital capacity in most patients. Moreover, in the few patients in whom these values do improve, the functional diagnosis is not modified.

\section{References}

1. Volta CA, Ploysongsang Y, Eltayara L, Sulc J, MilicEmili J. A simple method to monitor performance of forced vital capacity. J Appl Physiol 1996; 80: 693-698.

2. D'Angelo E, Carnelli V, D'Angelo E, Milic-Emili J. Performance of forced expiratory manoeuvre in children. Eur Respir J 2000; 16: 1070-1074.

3. Quanjer PH, Tammeling GJ, Cotes JE, et al. Lung volumes and forced ventilatory flows. Report Working Party Standardization of Lung Function Tests, European Community for Steel and Coal. Official Statement of the European Respiratory Society. Eur Respir Suppl 1993; 16: 5-40.

4. American Thoracic Society. Standardization of Spirometry, 1994 Update. Am J Respir Crit Care Med 1995; 152: 1107-1136.

5. D'Angelo E, Prandi E, Milic-Emili J. Dependence of maximal flow-volume curves on time course of preceding inspiration. J Appl Physiol 1993; 75: 1155-1159.

6. Bellia V, Pistelli R, Catalano F, et al. Quality control of spirometry in the elderly. Am J Respir Crit Care Med 2000; 161: 1094-1100.

7. Sherman CB, Kern D, Richardson ER, Hubert M, Fogel BS. Cognitive function and spirometry performance in the elderly. Am Rev Respir Dis 1993; 148: 123-126.

8. Carvalhaes-Neto $\mathrm{N}$, Lorino $\mathrm{H}$, Gallinari $\mathrm{C}$, et al. Cognitive function and assessment of lung function in the elderly. Am J Respir Crit Care Med 1995; 152: 1611-1615.

9. Langhammer A, Johnsen R, Gulsvik A, Holmen TL, Bjermer L. Forced spirometry reference values for Norwegian adults: the bronchial obstruction in NordTrondelag Study. Eur Respir J 2001; 18: 770-779.

10. Vanpee D, Swine C, Delwiche JP, Delaunois L. Evaluation of flow limitation in elderly patients unable to perform forced expiratory manoeuvre. Aging 2002 (in press).

11. Jones MH, Davis SD, Grant D, Christoph K, Kisling J, Tepper RS. Forced expiratory maneuvers in very young children. Assessment of flow limitation. Am J Respir Crit Care Med 1999; 159: 791-795. 